\title{
Infection Fatality Rate and Infection Attack Rate of COVID-19 in South American Countries
}

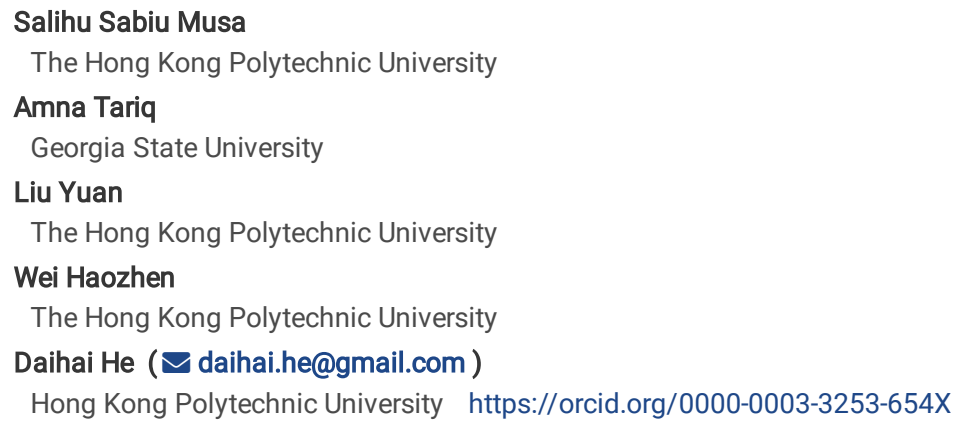

Keywords: COVID-19, epidemic model, infection attack rate, pandemic, reproduction number.

Posted Date: December 6th, 2021

DOI: https://doi.org/10.21203/rs.3.rs-1126392/v1

License: @ (i) This work is licensed under a Creative Commons Attribution 4.0 International License. Read Full License

Version of Record: A version of this preprint was published at Infectious Diseases of Poverty on April 6th, 2022. See the published version at https://doi.org/10.1186/s40249-022-00961-5. 


\section{Abstract}

Background: The ongoing COVID-19 pandemic hit South America badly with two waves. Different COVID-19 variants have been storming across the region, leading to more severe infections and deaths even in places with high vaccination coverages.

Methods: We use the start-of-the-art iterated filtering likelihood-based inference disease modelling framework. We modify the classical susceptible-exposedinfectious-recovered model with a time-varying transmission rate, and additional delayed class and vaccinations to reported COVID-19 deaths in 12 South American countries with the highest COVID-19 mortalities.

Results: We yield biologically reasonable estimates for the infection fatality rate (IFR), the infection attack rate (IAR) and time-varying transmission rate. We observe that the severity, the dynamical patterns of the deaths and the time-varying transmission rates among the countries are highly heterogeneous. Further, our analysis of the model with vaccination highlights that increasing the vaccination rate could effectively suppress the pandemics in South America.

Conclusion: This study reveals the possible mechanism behind the two waves of COVID-19 in South America. We observe reductions in the transmission rate corresponding to each wave plausibly due to improvement in nonpharmaceutical interventions (NPIs) measures and human protective behavior reaction to recent deaths. Thus, strategies coupling social distancing and vaccination could substantially suppress the mortality rate of COVID-19 in South America.

\section{Introduction}

The world has been facing devastating public health and socioeconomic growth problems ${ }^{1,2}$ in the ongoing COVID-19 pandemic. As of 29 September 2021 , the pandemic has caused more than 230 million cases and over 4.7 million deaths across the globe ${ }^{2}$. The pandemic hit China, Europe, North America first, then to rest part of the world, including the South and Latin America, Africa, and Western Pacific ${ }^{3-5}$. Guyana and Suriname are the two countries in South America that have recorded the lowest COVI-19 morbidity and mortality cases. By September 29, 2021, the mortality cases in these two countries were 792 and 893 , respectively ${ }^{6,7}$. Brazil and Peru are two of the hardest hit countries in terms of either total death cases or death cases per capita in South American region, with the subsequent waves heavily driven by new variants, e.g. P.1 variant $2,8,9$. By May 31,2020 , about $75.3 \%(4,196$ of 5,570$)$ of municipalities across all five administrative regions of Brazil reported COVID-19 cases, with 206,555 (40.2\%) recovery and 29,314 fatal (17.5\%) COVID-19 cases ${ }^{10}$. By July 28,2021 , the total death cases in Brazil and Peru were 553,272 and 196,138, respectively. In particular, Peru government updated the reported COVID-19 data and reported that $0.5 \%$ of the population died of the disease. This makes Peru and Brazil the first and seventh most affected countries in the world in terms of deaths per capita rate (https://coronavirus.jhu.edu/data/mortality). The situation in the Amazon region was extremely severe with high infection attack rates 10,11 .

The rate of COVID-19 vaccination is dramatically increasing worldwide, and the South American countries are scrambling to catch up by creating openings for vaccine diplomacy to reach the target for vaccinating at least $60-70 \%$ of the population. The region represents approximately $16 \%$ and $24 \%$ of the global cases and deaths by October 4, 2021. However, only about $7 \%$ of the worldwide vaccine doses have been administered in South America, according to a World Health Organization (WHO) report (2). By October 6, 2021, at least one dose of a COVID-19 vaccine has been administered to over $46 \%$ of the world population. And more than 6 billion doses of vaccines have been administered so far globally, with over 23.6 million administered each day (79), see Supplementary Table 1.

Although several effective vaccines are currently available ${ }^{12}$, yet, the nonpharmaceutical Interventions (NPIs) measures and other factors ${ }^{11,13}$ play significant roles in flattening the epidemic curves and help in reducing the mortality of COVID-19 across the globe. These measures include the changes in human behavior reaction, such as social distancing, usage of face masks ${ }^{11}$, possible pre-existing serological cross-reactivity against SARS-CoV-2 ${ }^{14}$, herd immunity 15 , availability of medical resources ${ }^{16}$, meteorological factors ${ }^{17,18}$, reduction in global transportation ${ }^{19,20}$, and usage of facemask ${ }^{21}$. These factors have resulted in highly geographical heterogeneity for COVID-19 transmission. Moreover, the spatiotemporal variability of COVID-19 epidemics has being studied across different levels through various indices ${ }^{22-24}$. Some countries in the South America, such as Chile, has greatly portrayed a positive impact following the NPIs and containment measures of COVID-19 implementation, which include localized lockdowns, banning of large gatherings, night-time curfew, and school and border closures ${ }^{25}$. These control measures likely help in suppressing the mortality cases in these countries, as well as preventing the epidemic trend from exponential increase especially during the early epidemic growth phase, as has happened in many countries across the globe ${ }^{25,26}$. The dynamic data dashboards (such as the Johns Hopkins data source of the IHME dashboard) of cases and deaths highlighted significant geographical variations in the epidemic patterns of COVID-19 worldwide $2,7,27$. Since the beginning of the pandemic, there has been a growing body of modeling studies to estimate the COVID-19 morbidity and mortality (see, for instance ${ }^{5,13,28-33}$ and the references therein). The transmissibility and severity of COVID-19 likely increased due to some devastating mutations, such as the $\mathrm{D} 614 \mathrm{G}$ amino acid. These evolved mutations may result from natural selection, and the steady increase of the $\mathrm{G} 614$ variant at regional stages could designate a fitness gain to this variant ${ }^{34,35}$. This mutation could increase the efficiency of the viral cell fusion to the host cell. Therefore, these variants have higher transmission rates ${ }^{36}$.

Small changes in the genetic code of viruses occur during the transmission. These changes are called "mutations". Most of the mutations are transient and some may persist in further transmission. The genetically modified versions of the virus with one or more new mutations from the original version is known as a "variant" 37 . According to the COVID-19 Genomic UK Consortium (COG-U.K.) ${ }^{38}$, there are thousands of COVID-19 mutations detected, but only few of them are likely to threaten public health ${ }^{39-41}$. Studies show that most viruses' mutations are not harmful and could not cause any severe infection ${ }^{40,42}$.

The P.1 variant with N501Y, E484K, and K417T mutations was detected on January 9, 2021, by the Japanese authorities in the airport from four travellers returning from Brazil ${ }^{43}$. Health authorities and epidemiologists are currently working to investigate whether this variant is more severe, besides its higher 
transmission rate, or could be a detriment to current therapies, diagnostics, or vaccines ${ }^{37,40}$. The P.1 lineage has been linked with high severity and reinfection scenario ${ }^{44-46}$. Previous report on SARS- CoV-2 genomic sequences highlighted that P.1 is more transmissible by up to 1.7 to 2.4 - fold and that previous infection by non- P.1 gives about $54-79 \%$ of the protection against P.1 infection compared with non- P.1 lineages ${ }^{47}$. Further information on the frequency and geographic distribution of the P1 lineage can be obtained at the Pango lineages website ${ }^{43}$. The mutations of this variant include the N501Y, which has some similarities with the variants identified in South Africa and the United Kingdom (UK) ${ }^{40}$. Currently, three COVID-19 variants are considered as the most dangerous variants that have raised public health concerns. These are lineage B.1.1.7 variant identified in the UK with N501Y mutation (which has now evolved to include the E484K mutation in UK) ${ }^{48}$; lineage B. 1.351 identified in South Africa; and the lineage P.1 variant identified in Brazil ${ }^{38,43}$. These variants are called "variants of concern (VOCs)" 40,49 . Since they potentially reduce antibody neutralization and increase affinity for ACE2 receptors, which results in increased severity and could lead to death. They are also linked to higher viral transmissibility, increased disease severity, and possible evasion of immunity, potentially impacting reinfection and vaccine effectiveness $9,42,50$.

The P.1 variant has been detected in over 70 countries ${ }^{43}$, including the United States, Canada, Belgium, Turkey, India, Brazil and Peru as of September 29 , 2021. It is currently storming across the South American region, giving rise to more severe cases and deaths even in places with high vaccination coverages. The resurgence of a second wave in the South America would be an important lesson for the rest of the world to tighten and improve the current control measures. Peru, a country of around 30 million people, is currently one of the world's hardest-hit countries with a COVID-19 mortality rate of about 200,000 (which makes the death per capita the highest globally by June 1, 2021) 7,51,52, and a resurgence observed in April 2021 that can be declared as the most deadly month for Peru since the pandemic began ${ }^{51,53,54}$. The flooded variant (P.1) were likely the cause of over $40 \%$ of infections in Lima ${ }^{54}$. Health authorities are also currently investigating another strain called C.37, which first emerged in Peru in August 2020, and has raised public health concerns, especially among the neighbouring countries such as Argentina, Chile, and Ecuador ${ }^{55}$. Colombia also experienced a similar scenario, where occupancy in intensive-care units hit $90 \%$ in the capital, Bogotá, and hospitals in other cities nearly overwhelmed ${ }^{54}$. A number of cross-sectional studies suggest that P. 1 variant is up to 2.2 times more contagious and as much as $61 \%$ more capable of reinfecting people than the original SARS-CoV-2 virus ${ }^{40,54,56,57}$.

Many countries in South America that experienced sharp rise in cases and deaths have, for the most part, not done extensive genomic sequencing to determine how many people have been infected by P. $1{ }^{9}$. Some reports show that the P.1 variant is the primary driver of the pandemic in the region ${ }^{9}$. Furthermore, according to Buss et al., the basic reproduction number $\left(R_{0}\right)$ for Amazonas was estimated at 2.5-3.0 ${ }^{10,11}$, which shows the high transmission potential of the virus to spread and cause large outbreaks. Moreover, the expected infection attack rate (IAR, i.e., proportion of the total population being infected) in a homogeneously mixed population during an unmitigated epidemic was estimated at $89-94 \%{ }^{58}$. When the percentage of infected individuals exceeds the herd immunity threshold of 60-67\% (which can be calculated using the relation $100 \times\left(1-1 / R_{0}\right)$ ) each infection generates less than one secondary case, thus incidence declines ${ }^{59}$. Estimation of vital epidemiological quantities, such as the infection fatality rate and reproduction numbers, are essential measures to understand the epidemics' spread to guide public health practitioners and policymakers in planning an effective and sustainable policy for disease prevention and control.

This study aimed to investigate spatiotemporal variability and similarity of the COVID-19 epidemic, as well as estimate IAR, IFR and reproduction number in the 12 most-affected countries in South America (i.e., Argentina, Bolivia, Brazil, Chile, Colombia, Ecuador, Guyana, Paraguay, Peru, Suriname, Uruguay, and Venezuela). Epidemiologically, the higher number of these parameters represents that the epidemic continues to spread. We also aimed to explore the transmission trends and identify the epidemic main drivers in the region (e.g., human behavioral changes, social distancing, and minimal or partial compliance of other NPIs measures by the general public). Further, we will compare the results of each scenario to reveal possible reasons for the current fluctuations (rise and fall) in mortality that would help to assess mitigation strategies, and inform public health responses and policymakers for effective control of the outbreak.

\section{Material And Methods 2.1 Epidemic data}

We retrieved the COVID-19 datasets from different sources. Epidemiological time-series datasets for COVID-19 cases and deaths are from publicly available reports collected and compiled by the WHO disease surveillance systems (dashboard) ${ }^{2}$. In particular, the data for Brazil was collected and compiled by the Brazil Ministry of Health disease surveillance report through the Secretariat Health Surveillance (SVS) available from ${ }^{60,61}$. Other datasets analyzed include: (i) state-level population data for Brazil ${ }^{62}$, (ii) state-level datasets for COVID-19 cases and deaths for Brazil ${ }^{63}$, (iii) state-level daily mortality datasets for Severe Acute Respiratory IIIness (SARI) obtained from hospitalized patients (which is a good proxy of COVID-19 deaths) available from ${ }^{64}$. SARG data (hospitalized SARI cases, first symptom onset date) ${ }^{65}$; and (vi) additional information on COVID-19 situation report for Peru were obtained from ${ }^{66}$.

Our analysis focuses on 12 most-affected countries with SARS-CoV-2 mortalities in South American region to estimate IFR, IAR and $R_{0}(t)$. For geographical locations of the 12 countries in South American region, see the map in Figure S3. The data used in the fitting processes covers all cases and deaths from 26 February 2020 until 2 January 2021. For each country the case incidence and mortality data were used. It is worth stating that, the current study did not assess the individual patient's data; hence no ethical approval and patient consent is required. Further, for Peru, we used COVID-19 death after Peru's official correction ${ }^{52}$. Consequently, the revised death in Peru is in line with "excess deaths" figures, which researchers have used in estimating the underascertainment of cases in Peru and other countries. Excess death accounts for the total number of extra deaths over time in comparison to the average level in the 5-year pre-pandemic period. For Brazil, we used the severe-acute-respiratory-infection-hospitalized deaths, which is larger than the official COVID-19 deaths 
cases and is believed to be a more accurate reflection of the actual scenario. We show the reported COVID-19 deaths and vaccination coverage over time in Figure 1.

\subsection{Epidemic model}

We used a modified susceptible-exposed-infectious-recovered (SEIR)-based model with a time-varying transmission rate $\beta(t)$ and a death class and a delay to death class. This model was used in previous work ${ }^{67,68}$. For the sake of completeness, we reintroduce it. We divided the total human population, $N(t)$ at time $t$ , into the mutually exclusive compartments of susceptible $S(t)$ (individuals who are at risk of the COVID-19 infection), exposed $E(t)$ (individuals who are exposed to COVID-19 infection), infectious (including asymptomatic, mild, and severe cases) $I(t)$, hospitalized severe cases $H(t)$, and recovered $R(t)$ individuals. And the compartment $D(t)$ accounts for the total number of people who dies due to COVID-19 infection. The model, represented in Figure 2, is given by the following system of differential equations.

$$
\begin{gathered}
\dot{S}=-\frac{\beta S I}{N}, \\
\dot{E}=\frac{\beta S I}{N}-\sigma E, \\
\dot{I}=\sigma E-\gamma I, \\
\dot{H}=\theta \gamma I-K H, \\
\dot{D}=\Pi K H, \\
\dot{R}=(1-\theta) \gamma I+(1-\Pi) K H .
\end{gathered}
$$

In the above equations, dot above the variables denotes the time differentiation. The parameter $\beta(t)$ represents the time-varying transmission rate, $\sigma$ is the infectiousness onset rate, $\gamma$ is the rate of loss of infectiousness, and $k$ represents the removal rate (due to death or recovery) of hospitalized cases. The parameter $\theta$ denotes the ratio of severe cases out of all infected cases and $\Pi$ represents the proportion of mortality out of severe cases. Hence, the overall CFR (or IFR) is equivalent to $\theta \Pi$. We note that the exactly definitions of $H, \theta$, and $\Pi$ are not important, since we only fit death data, rather than infected or hospitalized. Thus, the most important parameter is the IFR, i.e., the product $\theta \Pi$. In order to further simplify the model, we assume $\theta \approx \Pi$, thus the IFR is $\theta^{2}$. The possible reason for making this assumption is due to the unavailability of COVID-19 hospitalized severe cases data. We have tested alternatively where one of the two is fixed at some small values and yielded similar results. The class of $H$ serves as an intermediate delay class between infectives and deaths. We assumed all parameters of the model to be constant with the exception of the time-varying $\beta(t)$.

Following previous studies ${ }^{67-70}$, we define the transmission rate, $\beta(t)$, as an exponential cubic spline function ${ }^{71-73}$, i.e., $\beta(t)=\exp \left(c u b i c \_s p l i n e\right)$, with $n$ nodes evenly distributed over the study period, i.e., from 26 February 2020 to 24 June 2021 . We set time step size to be one day and integrated $\dot{D}$ for one day and yielded the simulated daily deaths $D_{t}$ We defined the reported deaths as $C_{t}$ which follows a negative binomial distribution

$C_{t} \sim$ NegativeBinomial $\left(\right.$ mean $=D_{t}$, variance $=D_{t}\left(1+\tau D_{t}\right)$ ),

where $\tau$ denotes the over-dispersion of reporting, and accounts for the measurement noise due to surveillance and heterogeneity among individuals. When $\tau=0$, this is reduced to a Poisson distribution. Namely, we assumed the reporting is an over-dispersed Poisson process, which is widely used and biologically reasonable.

Moreover, the following set of parameter values are used for the simulation results, that is, the mean latent period $(\sigma)$ as 2 days, the mean infectious period ( $\gamma$ ) as 3 days, and the mean duration of hospitalization $(K)$ as 8 days. We assumed that due to the time discretization in the simulation, the period should be slightly higher than these values. Thus, the sum of the mean latent period and infectious period are 6.07 days with a 1-day time step size (the sum approaches 5 days only when time step size approaches zero), which is close to the estimated generation time (GT, the sum of the mean latent and infectious period in an SEIR setting) by considerable amount of literature ${ }^{13,74-78}$. The mean duration from infection to death is about 14.57 days (with a 1 -day time step size), which is biologically reasonable ${ }^{79}$. We note that many previous studies used longer generation time, which is against the reported GT estimates of COVID-19 which may lead to overestimates of basic reproductive number ${ }^{78}$. We assumed that the initial susceptibility proportion is $95 \%$ to reflect the fact that a proportion of the population (e.g. children) are less susceptible ${ }^{80}$. We assumed that the initial infectious population is lower than 10,000 . The initial exposed and infectious populations are equal. The initial $H$ cases are 1/10 of the initial infectious cases. The initial deaths are $1 / 10$ of those of the $H$ cases. We fixed $n=9$ in the cubic spline. We assume $\theta$ between [0.055 and 0.085], thus IFR between [0.3\%, 0.72\%], see Figure 3. Similar results were respectively obtained for Figures S1 \& S2 with varying $\theta$, fixed $\Pi$, and different $n$. The estimates are roughly consistent. If we assume COVID-19 confirmed deaths are accurate, with estimated IFR, we can estimate the total infection in each country and the IAR.

\section{Results And Discussion}

In Figure 3, we depicted the fitting results for the top 12 South American countries. The time series of weekly confirmed COVID-19 deaths was denoted as red circle, the median of 1000 simulations was denoted as black curve, and the basic reproduction number is blue solidline, ie, $R_{0}(t)=\beta(t) / \gamma$. The shaded region denotes the $95 \%$ confidence region of 1000 simulations. We observed that there were disparities and similarities in the transmission rate across the 12 South American countries. 
Figure 3(a)-(I) have similarity, as can be seen from the simulation results. Each country is experiencing (or have experienced) at least two waves of COVID-19 epidemic with different time-varying effective reproduction number $\left(R_{0}(t)\right)$. The first peak of COVID-19 deaths reached around mid-October to November 2020 in Argentina, Bolivia, Brazil, Colombia, Guyana, Paraguay, Suriname, and Venezuela. While the first peak of COVID-19 deaths reached around July-August 2020, and the second peak reached around February 2021 in Chile, Ecuador, and Peru. By March-April 2021, the peak of the second wave in death cases is still in the process of descending in Argentina, Bolivia, Colombia, Suriname, and Uruguay, whereas the peak of the second wave is still in the process of increasing in Brazil, Chile, Ecuador, Guyana, Paraguay, Peru, and Venezuela. With The peak seems to be stabilizing at a level in Chile. The trends of COVID-19 in Brazil and Peru, the hardest hit countries (represented by panel (c) and (i) of Figure 3 ) have similarity. Based on the time-varying basic reproduction number, $R_{0}(t)$, each of these two countries show at least two-wave trends of COVID-19 mortalities, which is currently ongoing. The peak of deaths slightly follows the peak of $R_{0}(t)$. The slightly ascending $R_{0}(t)$ in Brazil and Peru around December 2020 predicted a rise in death cases within the next couple of weeks. Further, we observed a slight difference in the reproduction number across the cities of Brazil and Peru (see Figures S3 \& S4), which could be due to the differences in human behaviour, NPIs compliance, and availability of resources.

\subsection{Infection Fatality Rate}

We estimated the COVID-19 IFR for each of the 12 South American countries with the most deaths, as shown inside each panel of Figure 3 . The estimated IFR (or infection to reported death ratio) is shown inside each panel (a) to (I). The IFR varies between $0.303-0.723 \%$. Most countries in the region experienced similar trends of COVID-19 mortalities. Peru has been hit hardest, and, thus, have the highest IFR of $0.723 \%$. The IFRs were approximately similar for Brazil, Chile, Colombia, Paraguay, and Suriname, which are significantly higher than the IFR for the rest of the countries. Guyana has the lowest IFR estimated at $0.303 \%$, followed by Bolivia, Venezuela, Ecuador, Argentina, and Uruguay, which could be due to underreporting of deaths ${ }^{81}$. We note that our IFR is smaller than the reported raw case-fatality rates, since not all infections will be reported. For instance, according to Ramirez et al. ${ }^{82}$, the raw case fatality rate of COVID-19 in Argentina, Bolivia, Chile, Paraguay, and Brazil, by June 3, 2021, was estimated at 3.11\%, 3.42\%, 1.09\%, 1.09\%, 5.61\%, respectively 82 . Our estimated IFR for Brazil is lower than our estimated IFR in Manaus, Brazil ${ }^{59}$, which is the hit hardest region of Brazil. Further, a recent report by Rivera et al. ${ }^{83}$ revealed that the IFR for most countries in South America varies between $0.87-7.14 \%$ with a $95 \%$ confidence interval of $0.34-10.76 \%$.

\subsection{Infection Attack Rate}

Similarly, we estimated the corresponding IAR for each of the region's 12 countries, see Figure 3 (panel a-l). The IAR is shown in the title of each panel (a) to (I) (in bold font). The IAR varies between 0.03 to 0.784 . By June 2021, Peru is the hardest-hit country with COVID-19 mortality in terms of per capita rate. Peru has been hit hardest with an estimated IAR of $78.4 \%$, which means $78.4 \%$ of the population has been infected. The IAR for Peru was significantly higher than the rest of the South American region. Venezuela has the lowest IAR, which was estimated at 3\%, likely due to better compliance of COVID-19 containment measures, such as better social distancing policies and other human behaviour factors. While for the remaining countries, the IARs varies between 11.147.4\%. Most countries have also shown similar wave patterns in the region, especially as the second wave transmits faster in most countries.

\subsection{Assessing the effect of vaccination}

Here, the initial model was extended by incorporating the vaccination scenario. The COVID-19 vaccination rate $(v(t))$ represents the proportion of a population administered with a COVID-19 vaccine per day. The vaccination rate data were obtained from the WHO dashboard. The rate at which susceptible individuals get vaccinated is given by $\tilde{V}(t)=v(t) /\left(1-\int_{0}^{t-} v(s) \mathrm{ds}\right)$, where $\tilde{V}(t)$ represent the proportion of susceptible individuals who received a vaccine per day. We split the total population into two subpopulations, i.e., fully vaccinated and not fully unvaccinated groups. We target our analysis to not fully unvaccinated group with the second dose or first dose for previously infected.

The dynamic model is represented by the following system of nonlinear ordinary differential equations.

$$
\begin{gathered}
\dot{S}=-\frac{\beta S I}{N}-\eta \tilde{V} S, \\
\dot{E}=\frac{\beta S I}{N}-\sigma E, \\
\dot{I}=\sigma E-\gamma I, \\
\dot{H}=\theta \gamma I-K H, \\
\dot{D}=\Pi K H, \\
\dot{R}=\eta \tilde{V} S+(1-\theta) \gamma I+(1-\Pi) K H .
\end{gathered}
$$

The parameter $\eta=0.85$ denotes the proportion of the population that become fully protected over the study period. The model did not consider the reinfection scenario since it has been reported to be at a low rate and of mild symptom. Considering that the infection risk is not uniformly homogenous and some individuals have stronger immunity than others, we assumed that at least $5 \%$ of the population have a pre-existing cross-immunity from other coronaviruses $^{84}$. The initial exposed and infectious population were equal and randomly chosen in the range $[0,0.001]$ of the total population. $H$ class has $1 / 10$ of that in the infectious class, and Death (D) class has $1 / 10$ of that in the $H$ class.

We adopted a partially observed Markov process (POMP) model using maximum likelihood based iterated filtering technique to fit the mortality data 85 . It is worth mentioning that, the transmission rate $(\beta(t))$ was taken as an exponential cubic spline ${ }^{7172,73}$ to account for the simultaneous impact of the all-possible interventions including vaccination. For details on the fitting processes, see https://kingaa.github.io/sbied/. We analyzed the model "with" and "without" vaccination to evaluate the effect of the vaccination on the overall transmission dynamics. The model without vaccination was reconstructed by rerunning the fitted model with $v(t)=0$. Our model was the simplest model to explore vaccination's effect. However, one could incorporate the $V$ compartment to represent 
the proportion of the vaccinated population with low immunity response and susceptible to breakthrough infection. Our preliminary testing/analysis showed that these two models led to similar results.

\subsection{Strength and limitation}

In this paper, we chose one strain model rather than two strain model to explain the transmission patterns of SARS-CoV-2 in the South American countries, due to that fact that our death data are not strain specific. We assumed full cross-immunity between strains, and most parameter settings are considered to be constant and identical to two strains (e.g. wild strain and P.1 strain). Although two strains model could capture more dynamics and better explain the situation in South American region, but we considered one strain model and it works well with consideration of flexible transmission rate. Overall, our results suggested that the initial reproduction number of COVID-19 for hardest-hit countries (such as Brazil and Peru) were relatively high, likely due to social or environmental factors, i.e., low compliance of nonpharmaceutical interventions (NPIs) measures. Recall that during the ZIKV epidemics in South America, Brazil (especially the North-East) was hit much harder than the rest of the region. This scenario resembled some of the features of the current epidemics of SARS-CoV-2 in the region.

\section{Conclusion}

In summary, we developed a methodology to estimate the IFR and IAR of COVID-19 in South American countries. In particular, we used an SEIR type model with a time-varying flexible transmission rate. We observed that the initial reproductive number between countries in South America could be different, for some reasons (such as differences for NPIs compliance, healthcare standards, socioeconomic status, etc.). There were reductions in reproductive number (transmission rate) corresponding to each wave probably due to social distancing, human protective behavior reaction to recent deaths. This social distancing may be relaxed when the recent deaths decreased. This is more biologically reasonable than assuming the effective reproductive number as constant. This drop in reproductive number is not due to the depletion in susceptibility, since in our model we disentangled these two signals. Thus, this fluctuation in the transmission rate would lower the expected attack rate after each wave. Finally, our analysis of the model with vaccination indicated that increasing the vaccination rate could effectively suppress the pandemics in South America.

\section{Declarations}

\section{Ethics approval and consent to participate}

Ethics approval was not needed because all the data used can be found in the public domain.

\section{Consent to publish}

Not applicable.

\section{Availability of data and materials}

All the data used can be found in the public domain, available from https://covid19.who.int/.

\section{Competing interests}

We declared no competing interests.

\section{Funding}

This study was partially supported by a grant from the Research Grants Council of the Hong Kong Special Administrative Region, China (HKU C7123-20G).

\section{Authors' contributions}

Conceptualization: SSM \&DH; Formal analysis: SSM, AT, LY, WH, and DH; Writing - original draft: SSM, AT, LY, WH, DH; Writing - review \& editing: AT, DH.

\section{Acknowledgments}

We thank Prof. Gerardo Chowell-Puente for insightful discussion.

\section{References}

1. Hu, B., Guo, H., Zhou, P. \& Shi, Z.-L. Characteristics of SARS-CoV-2 and COVID-19. Nature Reviews Microbiology, 1-14 (2020).

2. World Health Organization (WHO). Coronavirus disease (COVID-19) Dashboard; 2020. https://covid19.who.int/. Accessed 10 Oct 2021., 2020).

3. Li, Q. et al. Early transmission dynamics in Wuhan, China, of novel coronavirus-infected pneumonia. New England journal of medicine (2020). 
4. Musa, S. S. et al. Estimation of exponential growth rate and basic reproduction number of the coronavirus disease 2019 (COVID-19) in Africa. Infectious diseases of poverty $9,1-6(2020)$.

5. Wu, J. T., Leung, K. \& Leung, G. M. Nowcasting and forecasting the potential domestic and international spread of the 2019-nCoV outbreak originating in Wuhan, China: a modelling study. The Lancet 395, 689-697 (2020).

6. Imperial College London. MRC Centre for Global Infectious Disease Analysis. Situation report for COVID-19: Suriname; 2021. https://mrcide.github.io/global-Imic-reports/SUR/. Accessed 9 July 2021., 2021).

7. Worldometer. COVID-19 Coronavirus Pandemic; 2021. https://www.worldometers.info/coronavirus/\#countries. Accessed 10 Oct 2021., 2020).

8. Naveca, F. et al. Three SARS-CoV-2 reinfection cases by the new Variant of Concern (VOC) P. 1/501Y. V3. (2021).

9. Naveca, F. G. et al. COVID-19 in Amazonas, Brazil, was driven by the persistence of endemic lineages and P. 1 emergence. Nature Medicine, 1-9 (2021).

10. de Souza, W. M. et al. Epidemiological and clinical characteristics of the COVID-19 epidemic in Brazil. Nature Human Behaviour 4, 856-865 (2020).

11. Buss, L. F. et al. Three-quarters attack rate of SARS-CoV-2 in the Brazilian Amazon during a largely unmitigated epidemic. Science 371, 288-292 (2021).

12. World Health Organization (WHO). COVID-19 Vaccines; 2021. https://www.who.int/emergencies/diseases/novel-coronavirus-2019/covid-19-vaccines. Accessed 1 Oct 2021., 2021).

13. Ali, S. T. et al. Serial interval of SARS-CoV-2 was shortened over time by nonpharmaceutical interventions. Science 369, 1106-1109 (2020).

14. Tso, F. Y. et al. High prevalence of pre-existing serological cross-reactivity against severe acute respiratory syndrome coronavirus-2 (SARS-CoV-2) in subSaharan Africa. International Journal of Infectious Diseases 102, 577-583 (2021).

15. Fontanet, A. \& Cauchemez, S. COVID-19 herd immunity: where are we? Nature Reviews Immunology 20, 583-584 (2020).

16. Moghadas, S. M. et al. Projecting hospital utilization during the COVID-19 outbreaks in the United States. Proceedings of the National Academy of Sciences 117, 9122-9126 (2020).

17. Ran, J. et al. The ambient ozone and COVID-19 transmissibility in China: a data-driven ecological study of 154 cities. The Journal of infection (2020).

18. Liu, J. et al. Impact of meteorological factors on the COVID-19 transmission: A multi-city study in China. Science of the total environment 726, 138513 (2020).

19. Du, Z. et al. Risk for transportation of coronavirus disease from Wuhan to other cities in China. Emerging infectious diseases 26, 1049 (2020).

20. Zhao, S. et al. The association between domestic train transportation and novel coronavirus (2019-nCoV) outbreak in China from 2019 to 2020 : a datadriven correlational report. Travel medicine and infectious disease 33, 101568 (2020).

21. Eikenberry, S. E. et al. To mask or not to mask: Modeling the potential for face mask use by the general public to curtail the COVID-19 pandemic. Infectious Disease Modelling 5, 293-308 (2020).

22. Castro, M. C. et al. Spatiotemporal pattern of COVID-19 spread in Brazil. Science 372, 821-826 (2021).

23. Chen, Y., Li, Q., Karimian, H., Chen, X. \& Li, X. Spatio-temporal distribution characteristics and influencing factors of COVID-19 in China. Scientific Reports 11, 1-12 (2021).

24. Xie, Z. et al. Spatial and temporal differentiation of COVID-19 epidemic spread in mainland China and its influencing factors. Science of The Total Environment 744, 140929 (2020).

25. Tariq, A. et al. Transmission dynamics and control of COVID-19 in Chile, March-October, 2020. PLoS Neglected Tropical Diseases 15, e0009070 (2021).

26. Gozzi, N. et al. Estimating the effect of social inequalities on the mitigation of COVID-19 across communities in Santiago de Chile. Nature communications 12, 1-9 (2021).

27. Johns Hopkins University of Medicine. Coronavirus Reseource Center, 02 Octorber 2021, <https://coronavirus.jhu.edu/> (2021).

28. Adam, D. C. et al. Clustering and superspreading potential of SARS-CoV-2 infections in Hong Kong. Nature Medicine 26, 1714-1719 (2020).

29. Lin, Q. et al. A conceptual model for the coronavirus disease 2019 (COVID-19) outbreak in Wuhan, China with individual reaction and governmental action. International journal of infectious diseases 93, 211-216 (2020).

30. Musa, S. S. et al. Mathematical modeling of COVID-19 epidemic with effect of awareness programs. Infectious Disease Modelling 6, 448-460 (2021).

31. Zhao, S. et al. Estimating the unreported number of novel coronavirus (2019-nCoV) cases in China in the first half of January 2020: a data-driven modelling analysis of the early outbreak. Journal of clinical medicine $\mathbf{9}, 388$ (2020).

32. Musa, S. S. et al. Estimation of COVID-19 under-ascertainment in Kano, Nigeria during the early phase of the epidemics. Alexandria Engineering Journal 60, 4547-4554 (2021).

33. Zhao, S. et al. Preliminary estimation of the basic reproduction number of novel coronavirus (2019-nCoV) in China, from 2019 to 2020: A data-driven analysis in the early phase of the outbreak. International journal of infectious diseases $92,214-217$ (2020).

34. Grubaugh, N. D., Hanage, W. P. \& Rasmussen, A. L. Making sense of mutation: what D614G means for the COVID-19 pandemic remains unclear. Cell 182, 794-795 (2020).

35. Zawbaa, H. M. et al. Effect of mutation and vaccination on spread, severity, and mortality of COVID-19 disease. Journal of Medical Virology (2021).

36. Chen, C.-Y., Chou, Y.-C. \& Hsueh, Y.-P. SARS-CoV-2 D614 and G614 spike variants impair neuronal synapses and exhibit differential fusion ability. bioRxiv (2020).

37. World Health Organization (WHO). The effects of virus variants on COVID-19 vaccines.; 2021. https://www.who.int/news-room/feature-stories/detail/theeffects-of-virus-variants-on-covid-19-vaccines. Accessed 29 Sep 2021, 2020).

38. COG-UK. COVID-19 Genomics UK Consortium, <https://www.cogconsortium.uk/> (2021).

Page $7 / 11$ 
39. Wise, J. (British Medical Journal Publishing Group, 2020).

40. Nguyen, K. V. Problems associated with antiviral drugs and vaccines development for COVID-19: approach to intervention using expression vectors via GPI anchor. Nucleosides, Nucleotides \& Nucleic Acids, 1-32 (2021).

41. Weisblum, Y. et al. Escape from neutralizing antibodies by SARS-CoV-2 spike protein variants. Elife 9, e61312 (2020).

42. Callaway, E. The coronavirus is mutating-does it matter? Nature 585, 174-177 (2020).

43. PANGO lineages. P.1;2021. https://cov-lineages.org/global_report_P.1.html. Accessed 10 Oct 2021, 2021).

44. Harvey, W. T. et al. SARS-CoV-2 variants, spike mutations and immune escape. Nature Reviews Microbiology 19, 409-424 (2021).

45. Naveca, F. et al. SARS-CoV-2 reinfection by the new Variant of Concern (VOC) P. 1 in Amazonas, Brazil. virological. org (2021).

46. Tang, X., Musa, S. S., Zhao, S. \& He, D. Reinfection or Reactivation of Severe Acute Respiratory Syndrome Coronavirus 2: A Systematic Review. Frontiers in Public Health 9 (2021).

47. Faria, N. R. et al. Genomics and epidemiology of the P. 1 SARS-CoV-2 lineage in Manaus, Brazil. Science 372, 815-821 (2021).

48. Public Health England. Investigation of Novel SARS-CoV-2 Variants: Variant of Concern 202012/01. Technical briefing 5.

https://assets.publishing.service.gov.uk/government/uploads/system/uploads/attachment_data/file/959426/Variant_of_Concern_VOC_202012_01_Tech Accessed 1 Sep 2021, 2021).

49. Geers, D. et al. SARS-CoV-2 variants of concern partially escape humoral but not T-cell responses in COVID-19 convalescent donors and vaccinees. Science Immunology 6 (2021).

50. Sabino, E. C. et al. Resurgence of COVID-19 in Manaus, Brazil, despite high seroprevalence. The Lancet 397, 452-455 (2021).

51. Dyer, O. (British Medical Journal Publishing Group, 2021).

52. Reuters. Healthcare \& Pharmaceuticals. Peru revises pandemic death toll, now worst in the world per capita.

https://www.reuters.com/world/americas/peru-almost-triples-official-covid-19-death-toll-after-review-180000-2021-05-31/. Accessed 20 June 2021., 2021).

53. Álvarez-Antonio, C. et al. Seroprevalence of anti-SARS-CoV-2 antibodies in Iquitos, Loreto, Peru. Loreto, Peru (2021).

54. TheWallStreetJournal. Brazil Covid-19 Variant Tears Through South America in Warning to World, <https://www.wsj.com/articles/brazil-covid-19-variantspreads-across-south-america-in-warning-to-world-11619611204> (2021).

55. National Geographic. Science Coronavirus Coverage. The unusual Lambda variant is rapidly spreading in South America. Here's what we know. Accessed 3 October 2021, <https://www.nationalgeographic.com/science/article/the-unusual-lambda-variant-is-rapidly-spreading-in-south-america-heres-what-weknow> (2021).

56. Brown, P. E. et al. Mortality from COVID in Colombia and Peru: Analyses of Mortality Data and Statistical Forecasts. medRxiv (2020).

57. Laiton-Donato, K. et al. Genomic epidemiology of SARS-CoV-2 in Colombia. medRxiv (2020).

58. Ma, J. \& Earn, D. J. Generality of the final size formula for an epidemic of a newly invading infectious disease. Bulletin of mathematical biology $68,679-$ 702 (2006).

59. He, D., Artzy-Randrup, Y., Musa, S. S. \& Stone, L. The unexpected dynamics of COVID-19 in Manaus, Brazil: Herd immunity versus interventions. medRxiv (2021).

60. Coronavirus. Brazil; 2021. https://covid.saude.gov.br/. Accessed 26 Oct 2021., 2021).

61. Amazonas. Brazil's Ministry of Health. Coronavirus Disease 2019. http://www.amazonas.am.gov.br/content/uploads/2021/01/20_01_21_BOLETIM_DIARIO_DE_CASOS_COVID-19-12.pdf. Accessed 10 Sep 2021, 2021).

62. Wikipedia. List of Brazilian states by population; 2021. https://en.wikipedia.org/wiki/List_of_Brazilian_states_by_population. 6 Jul 2021, 2021).

63. Wikipedia. Statistics of the COVID-19 pandemic in Brazil;2021. https://en.wikipedia.org/wiki/Statistics_of_the_COVID-19_pandemic_in_Brazil. Accessed $10 \mathrm{Jul} 2021,2021)$.

64. OpendataSUS. Brazil Ministério da Saúde Opendatasus SRAG $2020<\mathrm{https}$ ://opendatasus.saude.gov.br/dataset/bd-srag-2020> (2021).

65. OpendataSUS. SRAG 2020 - Severe Acute Respiratory Syndrome Database - including data from COVID-19, <https://opendatasus.saude.gov.br/gl/dataset/bd-srag-2020/resource/d89ea107-4a2b-4bd5-8b8b-fa1caaa96550> (2021).

66. Wikipedia. Template:COVID-19 pandemic data/Peru medical cases; 2021. https://en.wikipedia.org/wiki/Template:COVID19_pandemic_data/Peru_medical_cases\#cite_note-258. Accessed 10 Oct 2021, 2021).

67. Song, H., Fan, G., Liu, Y., Wang, X. \& He, D. The second wave of COVID-19 in South and Southeast Asia and vaccination effects. doi:DOI: 10.21203/rs.3.rs877949/v1 (2021).

68. Song, H. et al. Forecast of the COVID-19 trend in India: a simple modelling approach. doi:DOI: 10.21203/rs.3.rs-502990/v1 (2021).

69. He, D., lonides, E. L. \& King, A. A. Plug-and-play inference for disease dynamics: measles in large and small populations as a case study. Journal of the Royal Society Interface 7, 271-283 (2010).

70. Zhao, S., Stone, L., Gao, D. \& He, D. Modelling the large-scale yellow fever outbreak in Luanda, Angola, and the impact of vaccination. PLoS neglected tropical diseases 12, e0006158 (2018).

71. Bartels, R. H., Beatty, J. C. \& Barsky, B. A. An introduction to splines for use in computer graphics and geometric modeling. (Morgan Kaufmann, 1995).

72. Vetterling, W. T., Press, W. H., Teukolsky, S. A. \& Flannery, B. P. Numerical recipes: example book C (The Art of Scientific Computing). (Press Syndicate of the University of Cambridge, 1992). 
73. Burden, R. L. \& Faires, J. D. Numerical analysis 8th ed. Thomson Brooks/Cole (2005).

74. Ferretti, L. et al. Quantifying SARS-CoV-2 transmission suggests epidemic control with digital contact tracing. Science 368 (2020).

75. He, X. et al. Temporal dynamics in viral shedding and transmissibility of COVID-19. Nature medicine 26, 672-675 (2020).

76. Zhao, S. Estimating the time interval between transmission generations when negative values occur in the serial interval data: using COVID-19 as an example. (2020).

77. Zhao, S. et al. Estimating the serial interval of the novel coronavirus disease (COVID-19): A statistical analysis using the public data in Hong Kong from January 16 to February 15, 2020. (2020).

78. Tang, X., Musa, S. S., Zhao, S. \& He, D. Using proper mean generation intervals in modelling of COVID-19. Frontiers in Public Health, doi:10.3389/fpubh.2021.691262 (2021).

79. Baud, D. et al. Real estimates of mortality following COVID-19 infection. The Lancet infectious diseases 20, 773 (2020).

80. Rozhnova, G. et al. Model-based evaluation of school-and non-school-related measures to control the COVID-19 pandemic. Nature communications 12 , $1-11$ (2021).

81. Paniz-Mondolfi, A. E., Sordillo, E. M., Márquez-Colmenarez, M. C., Delgado-Noguera, L. A. \& Rodriguez-Morales, A. J. The arrival of SARS-CoV-2 in Venezuela. The Lancet 395, e85-e86 (2020).

82. Ramírez, M. L., Martinez, S. M., del Valle Bessone, C., Allemandi, D. A. \& Quinteros, D. A. COVID-19: epidemiological situation of Argentina and its neighbor countries after three months of pandemic. Disaster medicine and public health preparedness, 1-23 (2021).

83. Simbana-Rivera, K. et al. Interim analysis of pandemic Coronavirus disease 2019 (COVID-19) and the SARS-CoV-2 virus in Latin America and the Caribbean: morbidity, mortality and molecular testing trends in the region. MedRxiv (2020).

84. Ng, K. W. et al. Preexisting and de novo humoral immunity to SARS-CoV-2 in humans. Science 370, 1339-1343 (2020).

85. Ionides, E. L., Bretó, C. \& King, A. A. Inference for nonlinear dynamical systems. Proceedings of the National Academy of Sciences 103, $18438-18443$ (2006).

\section{Figures}
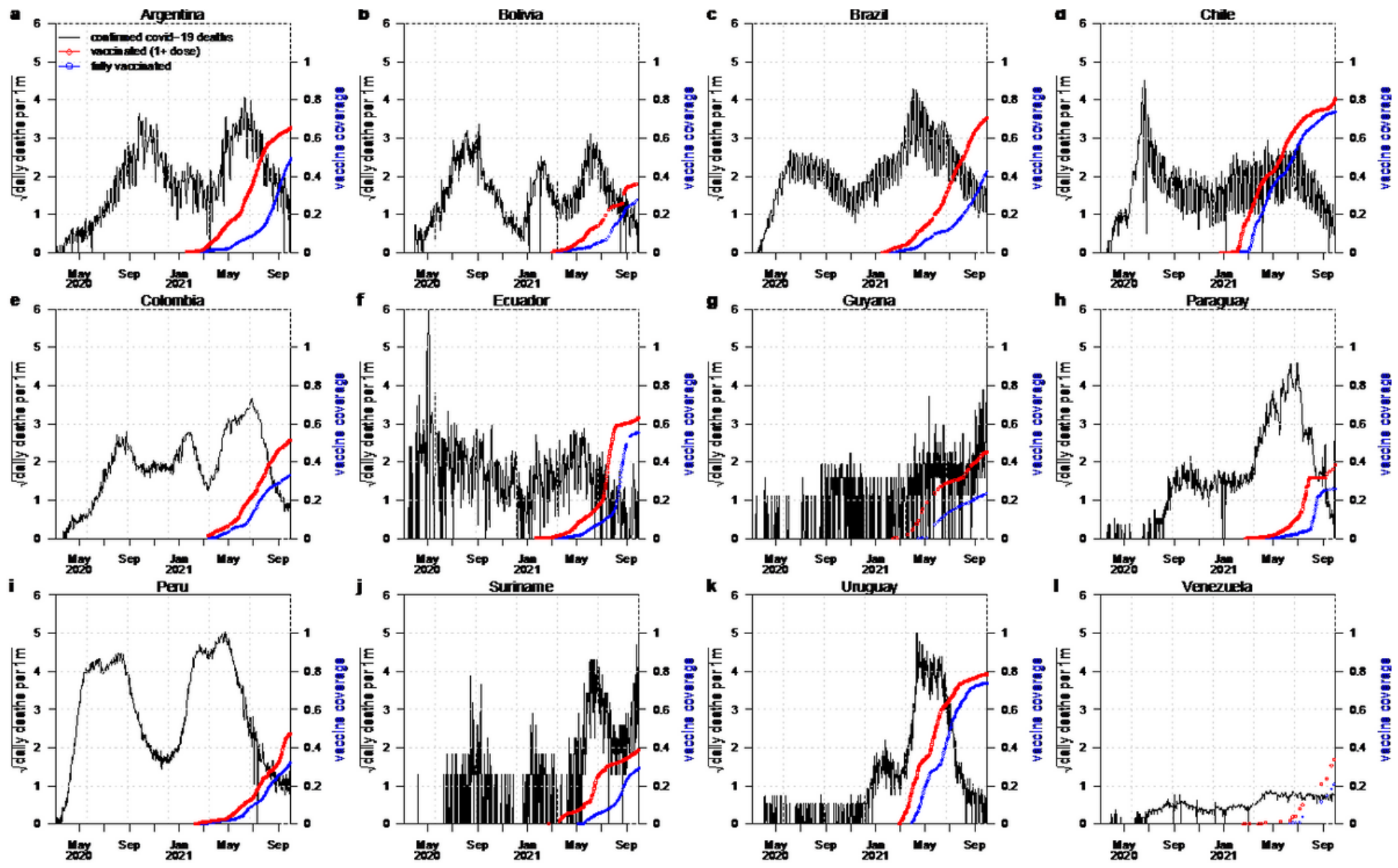

Figure 1

The reported deaths per million population (black curve, in square root scale such that we can see the small values) for 12 Southern American countries, and the vaccination coverage partly one-dose vaccinated and fully two-dose vaccinated, in red and blue curve, respectively. 


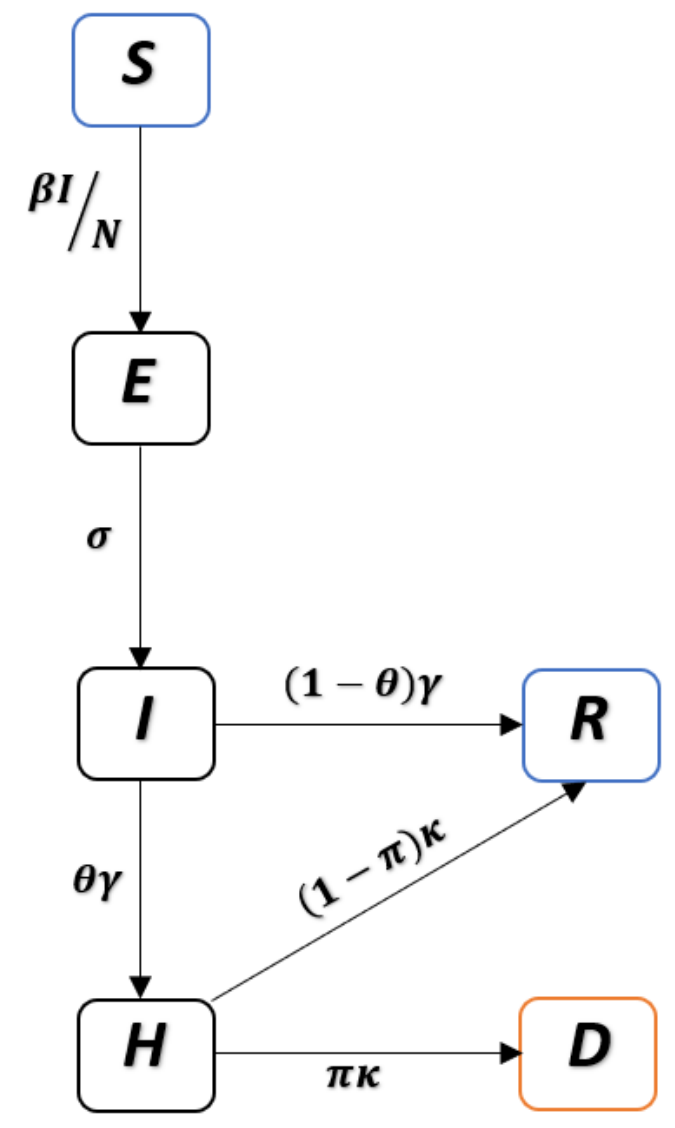

Figure 2

Schematic diagram of COVID-19 model without vaccination. 

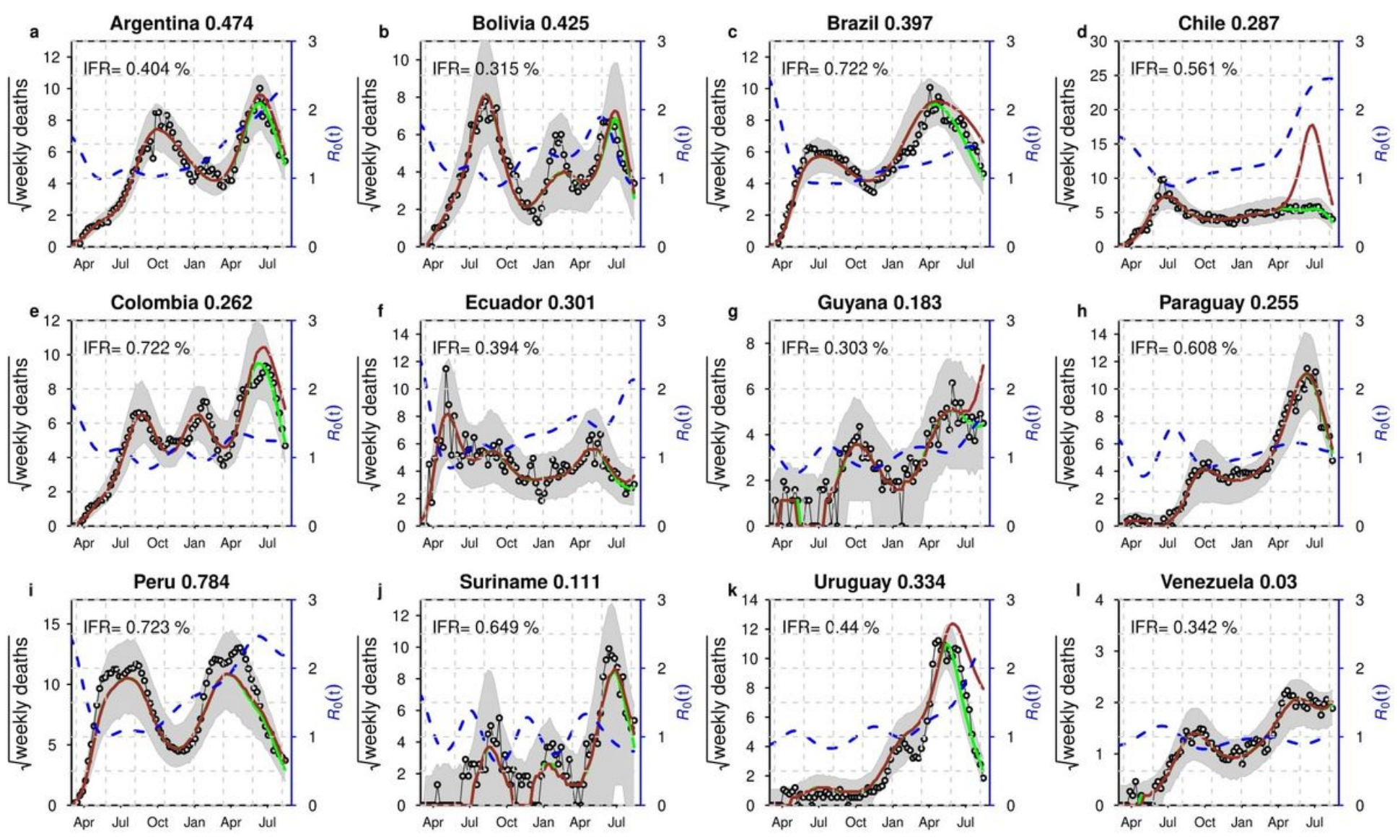

Figure 3

Fitting results for the twelve South American countries with highest reported COVID-19 deaths (represented by panels a-ls). Time series plots for the weekly reported COVID-19 deaths (in red circle) with simulation median (in black) and the basic reproduction number, R_0 (t), in solid blue line. The shaded regison represents the $95 \%$ confidence interval of the simulation. The numbers from each panels a-o represent the IAR for the 12 countries, respectively. With $\pi=\theta$, and $n=9$. The resurgence of deaths in Brazil and Peru could be explained by resurgence of R_0 ( $t$ ) due to emergence of new variant and relaxing of social distancing.

\section{Supplementary Files}

This is a list of supplementary files associated with this preprint. Click to download.

- 211130 suplementarymaterial.docx 